\section{TARA study: a new perspective on tapering drugs in RA}

We read with great interest the article on 'Gradual tapering TNF inhibitors vs conventional synthetic DMARDs after achieving controlled disease in patients with rheumatoid arthritis: firstyear results of the randomised controlled TARA study ${ }^{1}$ by van Mulligen et al. This was the first head-to-head comparison between two tapering strategies-biological versus conventional in rheumatoid arthritis. The final results favour tapering tumour necrosis factor inhibitors (TNFis) before conventional synthetic disease-modifying antirheumatic drugs (csDMARDs). However, certain points need clarifications.

First, many patients in the study used combination csDMARDs, but no data have been provided on their number and specific combinations used. Furthermore, no clarity has been given on how the tapering was done in these patients who were on combination csDMARDs-was methotrexate the only drug reduced and stopped or were all the drugs in the combination reduced and stopped. In their trial registration (NTR2754), the authors have only mentioned methotrexate tapering, whereas the study title mentions csDMARD tapering.

Second, in their statistical analysis, they mention an 'intentionto-treat analysis'. However, in their results, the authors have presented the clinical response after 12 months for both tapering groups, for only 85 and 89 patients in table 2 (final number of patients at 12 months) rather than the 94 and 95 patients who were initially randomised to the two tapering arms.

Third, the number of patients in clinical remission (disease activity score (DAS) <1.6) in TNFi tapering arm has been reported in table 2 as 58 . However, the number of patients at risk at 12 months has been mentioned as only 54 (figure 2B). Thus, it is unclear that how the number of patients in clinical remission at 12 months more than the number at risk-ideally, all patients who dropped out or required to restart biologics or csDMARDs because of flare should be not considered in remission.

Finally, in the study protocol, the use of intra-articular glucocorticoids (GCs) and one intramuscular (IM) injection of GCs during a flare (as bridging therapy) was permitted. However, four and five patients, respectively, in csDMARDs and TNFi tapering arms received oral GCs and three patients in each arm got more than one IM injections. What was the effect of excluding these patients on the analysis would be interesting to know?

Debashish Mishra ๑ , Arghya Chattopadhyay ๑ , Sanjay Jain, Varun Dhir Clinical Immunology and Rheumatology services, Department of Internal Medicine, PGIMER, Chandigarh, India

Correspondence to Dr Varun Dhir, Internal Medicine (Rheumatology Unit), Post Graduate Institute of Medical Education and Research, Chandigarh 160012, India; varundhir@gmail.com

Contributors All authors (DM, AC, SJ and VD) were involved in the preparation of the manuscript and reviewed the final version.

Competing interests None declared.

Patient consent for publication Not required.

Provenance and peer review Not commissioned; internally peer reviewed.

(c) Author(s) (or their employer(s)) 2020. No commercial re-use. See rights and permissions. Published by BMJ.

\section{D) Check for updates}

To cite Mishra D, Chattopadhyay A, Jain S, et al. Ann Rheum Dis 2020;79:e79.

Received 23 April 2019

Accepted 25 April 2019

Published Online First 9 May 2019

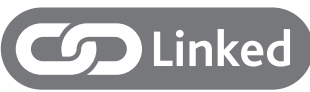

- http://dx.doi.org/10.1136/annrheumdis-2019-215641

Ann Rheum Dis 2020;79:e79. doi:10.1136/annrheumdis-2019-215594

ORCID iDs

Debashish Mishra http://orcid.org/0000-0001-8411-2015

Arghya Chattopadhyay http://orcid.org/0000-0002-3626-0694

\section{REFERENCE}

1 van Mulligen E, de Jong PHP, Kuijper TM, et al. Gradual tapering TNF inhibitors versus conventional synthetic DMARDs after achieving controlled disease in patients with rheumatoid arthritis: first-year results of the randomised controlled tara study. Ann Rheum Dis 2019;78:746-53. 